\title{
Relative selection as a function of two pre-weighted criteria: A study of two procedures
}

\section{Louis Laurencelle $\stackrel{\Xi}{ }$, a}

a Département des Sciences de l'activité physique, Université du Québec à Trois-Rivières

\begin{abstract}
In contrast with absolute selection, which retains any candidate reaching some pre-determined level of the selection variables, relative selection deals with a pool of candidates among which a given number of individuals must be recruited. Trivially done when only one criterion is to be used, implementation of relative selection becomes more involved when two or more criteria are applied, each criterion having its own a priori, predetermined weight and no outcome measure being available. For instance, with two criteria, how may information from both criteria be combined, and can the respective, a priori weights assigned to the criteria be enforced on the selection results? The problem is analysed, and tentatively solved, and two implementation schemes, - additive and sequential - are described, mathematically examined, and exemplified.
\end{abstract}

Keywords " Relative selection, composite score, pre-weighted criteria, nominal weights, additive scheme, sequential scheme, multiple-hurdle approach

$\equiv$ louis.laurencelle@gmail.com

\section{Introduction}

Personnel selection systems used for qualifying candidates for a job or for sorting out the most worthy individuals from a group of contenders operate in a diversity of modes (Barrett et al., 1978; Dunnette \& Borman, 1979), ranging from qualitative, one-person judgements up to data-based classification algorithms, such systems being either absolute or relative. An absolute, or minimum qualification, selection system is one in which the merit of each candidate is appraised on the basis of some qualification criterion or criteria, compared to pre-determined standard or valuethreshold, and then decided upon, independently of any remaining candidates. Relative selection, by contrast, considers all competing candidates at once and compares their merits from one to the other, thus highlighting a number of persons agreed to in advance and retaining them, independently of any externally defined threshold. In this essay, we consider relative selection systems based on two pre-weighted quantitative criteria, and we investigate two distinct schemes of implementation, an additive scheme using a composite, or weighted sum, of selection variables, and a sequential or multi-stage scheme in which the selection variables are applied sequentially.

Many selection systems can be, and have been, thought up, applied and compared (e.g., Sackett \& Roth,
1996), as can be seen in recent reviews on the subject (Guion, 2011; Ployhart, 2012). Documented and published systems pertain mostly to job selection contexts and they aim at predicting in some way the future 'output' of the candidate or some variable thereof; for systems using two or more predictive variables and an output measure, multiple regression analysis (Schmidt \& Hunter, 1998) is a standard method, providing the user with a weighted composite of the predictors, optimally related to the target variable $^{1}$. Although "success-at-the-job" remains an important asset for selection, other views are

\footnotetext{
${ }^{1}$ In the psychometric literature (e.g. Cronbach, 1971; Kane, 2006), predictive selection systems are dealt with under the headings of predictive validity and criterionbased validation, i.e. validation of a test score by its correlation with or regression on some recognized 'criterion', frequently an outcome or 'output' variable. The term 'criterion' in that context is thus reserved for the outcome variable. In the present article, however, no reference is made to any outcome or outcome variable nor to any external validation of the selection results. This allows us to use the term 'criterion' more broadly, with the connotation that 'criterion' is the psychometric construct for which the corresponding 'selection variable' stands for.
}

The Quantitative Methods for Psychology 
legitimate, for instance judgementally-based selection decisions, inasmuch as their operation is based 'on some logical, empirical and theoretical foundation' (SIOP, 1987). A quite common example of such a logical / theoretical evaluation approach is selection of candidates for an academic or professional training program. For instance, for would-be physicians, teachers or plumbers, there may be no readily available measures of 'professional success', and only the skills and knowledge obtained and measured during academic training can be considered. Moreover, 'success-at-the-program', whether available or not, may not be a sufficient or even an admissible variable for foretelling a decent practitioner. As noted by Deckro (1977), any single measure of performance or academic output, be it simple or composite, may pass by crucial requisites, such as technical or interpersonal know-how and moral standing, qualities to which careful interviewing and qualitative appraisal can hopefully attest.

Hence, for any combination of the above reasons, organizations have set up selection systems based on two or more a priori criteria, each being assigned a "nominal" weight: for instance, for recruiting and hiring factory workers, a classification system could be based on "working ability" for $80 \%$ and "honesty" for $20 \%$, the first component measured by a standardized, onsite test protocol, and the second, by an "honesty test" (Ones, Viswervaran and Schimdt, 1993) or through psychological interviewing. Murphy and Shiarella (1997) discuss such systems. Moreover, Guion (2011) recommends them, in these words:

A weighting scheme matters if only a few variables form the composite. In these cases, it should be based on rational or theoretical grounds rather than on computation alone... [In either case,] it is wise to see if the effective weights make sense. [Guion, 2011, p. 276-277].

We must open a parenthesis to further define and clarify our concept of "nominal weights", which pertain here to numbers purported to express the relative importance of a set of criteria and are imposed by decree by some governing authority. "Nominal weights" appear in another, somewhat different guise in the literature (Wang \& Stanley, 1970; Kolen 2004; Guion, 2011), where they denote simple coefficients in a linear composite (e.g., $b_{X}$ and $b_{Y}$ in $b_{X} \cdot X+b_{Y} \cdot Y$ ), in contrast with "effective weights" which reflect the contribution in variance of each linear component, including its proper variance and its possible covariances shared with other components. Moreover, our experimental results show that the two definitions are not equivalent. We feel that our use of the verbal label 'nominal weight' is unavoidable, and pray the reader to indulge us and keep in mind the particular, and more natural, definition that we propose.

For systems based on numerous, positively correlated variables, it has been shown that all positive-weighted composites are about statistically equivalent: unit-weights, random positive weights, rational weights and regression-based weights composites all correlate highly, with correlation coefficients near 1 (Wang and Stanley, 1970; Dawes and Corrigan, 1974; Cronbach, 1990; Guion, 2011). Of course, this high level of correlation between various positive-weighted composites crumbles down as the number of linear components decreases. This actual quasi equivalence of linear composites leaves room for adopting rational weights and rationally accountable weighting rules and principles, which will be easier to handle and legitimize in cases of dispute (Guion, 2011).

Most selection systems reviewed in the foregoing literature refer, explicitly or implicitly, to absolute selection, even if authors (e.g., Cronbach, 1990) allude to relative selection when describing situations where a pre-determined number of candidates must be retained. In spite of this, we were able to find but a few papers dealing with relative selection and its mechanisms, e.g., Sackett and Roth (1996)'s where 14 different selection rules on two criteria are compared through Monte Carlo simulations, and none regarding relative selection based on two nominal-weighted criteria, used either in an additive or a sequential implementation scheme. This is not to say that such selection systems are imaginary or their use unprecedented. On the contrary, on the basis of our own experience, we feel assured that relative selection is quite common, even commoner (and cheaper!) than absolute selection, at least in small and medium-sized enterprises and limited enrolment training programs such as for medicine and teaching. Among those organizations, there are some who use two or more differentially weighted criteria embedded either in the "additive" or the "hierarchical" scheme, as we call them: how are the weights integrated into the selection scheme, and how can one assess the correspondence between the weights' values and the selection results? Let's now posit the problem, clarify the issues and present some preliminary results. 


\section{Two pre-weighted criteria, and does the output matches the input?}

Plainly stated, relative selection amounts to the eventual recruitment and hiring of the best $n$ among $\mathrm{N}$ postulants. With only one criterion and corresponding selection variable, relative selection is trivially carried out: the $N$ candidates are arranged in descending order as a function of their value on the variable, the individuals to be retained being those appearing in the $n$ positions at the top of the list. With two criteria/variables or more, the problem becomes more involved, at two levels: first, the criteria need be weighted (unless equal weights are appropriate for a particular situation and are agreed to), and, second, the selection scheme must ensure that each criterion's predetermined weight is duly transferred into the selection results. For instance, let's suppose a manufacturing plant operates a selection system based on two measures, 'manual ability' and 'honesty', respectively weighted $80 \%$ and $20 \%$ : how should we combine information form the two measures? One may naïvely propose an additive combination such as:

$$
T=0.80 \times(\text { Ability score })+0.20 \text { (Honesty score }),
$$

then selecting the wished for number of people who obtain the highest $T$ values. What is the real outcome of this selection rule, and how do the results, i.e. the array of persons retained, match the imposed 80/20 weights? In fact, as we shall see, the deployment of results (e.g., how many candidates were favoured by each criterion) depends in a complex manner on each score's variance, their mutual correlation, and even on the selection rate. A naïve formula such as the one above could well lead to absurdly irrelevant results.

For the sake of clarity, the following developments will be limited to the case of two criteria, although most results and conclusions are easily extended to criteria in any number.

\section{Origin of the weights}

Where do the weights come from? The weights are usually conceived as to reflect the relative importance of the criteria they stand for, and they can emanate from a careful analysis of the situation, from a global appreciation of the profile of the wished for candidates, or even from available statistical information such as previous multiple regression analyses. The recruitment of factory workers broached earlier, with a weight of
$80 \%$ for 'working ability' and $20 \%$ for 'honesty', is a paradigmatic instance, as that of selecting candidates for enrolment in an academic training program based on the GPA score for $70 \%$ and 'interpersonal skills' for $30 \%$ as quantitatively appraised through a standardized group interview. In any case, at some point in time prior to the selection process, some legislative body, a committee, an administrator has defined the criteria and decreed their respective weights, say $w_{X}$ and $W_{Y}$, expecting that such weights be proportionally implemented in the selection results.

\section{Rescaling the weights}

The weights $\left(w_{i}\right)$ assigned to the criteria are, by definition, positive $\left(W_{i}>0\right)^{2}$ and they can be rescaled so that they sum up to 1 , i.e. $\Sigma W_{i}=1$. Take, for instance, a 2-criterion system with weights $w_{X}=0.75$ and $w_{Y}=$ 0.25 , meaning in a broad sense that criterion $X$ should have an influence three times as large as criterion $Y$ on the selection results. But, how can we relate the decreed, predetermined weights, on the one hand, to the pattern of selection results on the other?

\section{The dominance index}

Let the two criteria be embodied by selection variables $X$ and $Y$, with corresponding weights $w_{X}$ and $w_{Y}$. From an initial pool of $N$ candidates, the selection process (to be studied in the following sections) shall produce a list of $n$ chosen recruits. Now, the $n$ elements in the final list of selected candidates have been spotted and retained as a function of both variable $X$ and variable $Y$, but this does not entail that each chosen candidate would have separately been qualified both on $X$ and on $Y$, or even on any one variable. More precisely, the $n$ elements forming the final list of selected individuals may be subdivided in the following four categories,

$n_{X, Y}$ : elements (candidates) who would have been retained either by variable $X$ or $Y$;

$n_{X}$ * elements who would have been retained by variable $X$ but not variable $Y$;

$n^{*}, Y_{*}$ elements who would have been retained by variable $Y$ but not variable $X$;

$n * *$ : elements who would have been retained neither by variable $X$ or $Y$.

Indeed, to explain the $n^{*}$, component, a candidate may be retained by the selection system even if he/she

2 Because they reflect the relative 'importance' of the criterion. They are not to be confused with linear regression weights, which can have any real value. 
Table 1 - Comparing the $D_{X}$ and $\operatorname{Pr}_{X}$ indices for various parametric conditions for the selection of $n$ individuals among $N=1000$ candidates, under the additive selection scheme ${ }^{t, *}$

\begin{tabular}{cccccc}
\hline \multirow{2}{*}{$N$} & \multirow{2}{*}{$b_{X}$} & \multicolumn{2}{c}{$\rho_{X Y}=0$} & \multicolumn{2}{c}{$\rho_{X Y}=0.5$} \\
\cline { 3 - 6 } 100 & 0.1 & 0.039 & 0.071 & 0.071 & 0.218 \\
& 0.3 & 0.144 & 0.143 & 0.234 & 0.309 \\
& 0.5 & 0.296 & 0.278 & 0.423 & 0.441 \\
& 0.7 & 0.495 & 0.494 & 0.635 & 0.601 \\
& 0.9 & 0.759 & 0.773 & 0.869 & 0.754 \\
\hline \multirow{3}{*}{200} & 0.1 & 0.051 & 0.126 & 0.077 & 0.275 \\
& 0.3 & 0.171 & 0.203 & 0.244 & 0.353 \\
& 0.5 & 0.316 & 0.324 & 0.424 & 0.455 \\
& 0.7 & 0.491 & 0.493 & 0.625 & 0.575 \\
& 0.9 & 0.733 & 0.721 & 0.860 & 0.699 \\
\hline \multirow{4}{*}{300} & 0.1 & 0.059 & 0.178 & 0.082 & 0.319 \\
& 0.3 & 0.186 & 0.252 & 0.248 & 0.383 \\
& 0.5 & 0.326 & 0.355 & 0.425 & 0.464 \\
& 0.7 & 0.494 & 0.496 & 0.627 & 0.560 \\
& 0.9 & 0.722 & 0.681 & 0.856 & 0.659 \\
\hline
\end{tabular}

$\dagger \rho_{X Y}$ is the value of the linear correlation coefficient used for generating each list of $(X, Y)$ values for the $N$ candidates.

* Score applied for selecting selecting is $b_{X} \cdot X+b_{Y} \cdot Y$ (variables $X$ and $Y$ being standardized), where $b_{\mathbf{Y}}=\sqrt{\left(b_{X} \rho_{\mathrm{XY}}\right)^{2}+1-b_{X}^{2}}-b_{X} \rho_{\mathrm{XY}}$

would have failed to rank among the $n$ best scores in both his/her $X$ and his $Y$ variables, their joint values having "pushed" him (i.e. additively) to success.

Among the $n$ elements retained, those $\left(n_{X, Y}\right)$ who would have qualified on both criteria and those $(n *$,$) on$ neither one render no information about the relative importance of each criterion ; only the discordant counts, $n_{X^{*}}$ and $n^{*}, Y$, do inform us about it. Based on these data, the dominance index for criterion $X\left(D_{X}\right)$ is defined by:

$$
D_{X}=n_{X^{*}} /\left(n_{X^{*}}+n_{*, Y}\right),
$$

i.e. the proportion of discordant decisions favouring criterion $X$, the dominance for $Y$ being defined likewise, with corollary $D_{Y}=1-D_{X}$. For instance, $D_{X}=1$ reflects a situation where, apart the $\left(n_{X, Y}\right)$ candidates elected both on the $X$ and $Y$ variables, the remaining selected candidates were all favoured by the $X$ criterion, such an utopian case corresponding to a $w_{X}=1, w_{Y}=0$ weight pair.

\section{The precedence index}

Another way to look at the selection data, composed of $n(X, Y)$ values, is to compare the relative ranks of selected candidates on $X$ and $Y$. For instance, if criterion
$X$ should dominate (e.g., with $w_{X}=0.90$ and $w_{Y}=0.10$ ), one would expect that, on the average, the $X$-rank for a candidate exceed his $Y$-rank, the ranks being established from the original full list of $N$ candidates. In other words, the relative import of the $X$ criterion should reflect on the ranks of the selected individuals, their $X$-rank values preceding, i.e. being higher than, a number of the $Y$-rank values. The precedence index of $X$ is then defined by:

$\operatorname{Pr}_{\mathbf{X}}=\frac{\sum_{i, j}^{n} r\left(X_{i}\right)>r\left(Y_{j}\right)+0.5 \sum_{i, j}^{n} r\left(X_{i}\right)=r\left(Y_{j}\right)}{n^{2}}(2)$

where $r\left(X_{i}\right)$ is the rank (numbered 1 to $N$ ) of candidate $i$ for his $X$ score among the $N X$ values, etc. , and $0 \leq \operatorname{Pr}_{X}$ $\leq 1$. The precedence of $Y$ is defined likewise, with $\operatorname{Pr}_{Y}=$ $1-\operatorname{Pr}_{X}$

We ran a series of Monte Carlo experiments in order to assess the comparative merits of the $D_{X}$ and $\operatorname{Pr}_{X}$ indices, using the additive selection scheme (to be explained later). Table 1 is illustrative of our results.

Summarily, the two indices are equally responsive to variations of the criteria's weights, and both are sensitive to the selection ratio $(n / N)$ and correlation $\left(\rho_{X Y}\right)$ between criteria. Moreover, Spearman's rankorder correlation between $D_{X}$ and $\operatorname{Pr}_{X}$ borders on 1 . However, as can be seen in Table 1 , the $D_{X}$ index tends 
to show a broader range of values, and its interpretation for the layman seems plainer. For all practical purposes, we will use the dominance index, $D_{X}$

\section{Selection by the sum of the two variables}

The additive scheme of selection essentially consists in adding in some way the two criterion-related variables for every candidate, then deciding on the basis of the obtained totals. In the course of the selection process, each candidate is given an $X$ and a $Y$ value, their sum ( $T$ ) being computed as:

$$
T=B_{X} \cdot X+B_{Y} \cdot Y+C,
$$

$B_{X}$ and $B_{Y}$ being appropriate weighting coefficients, and $C$ an arbitrary constant. This additive utilization of two or more selection variables for determining the status or rank of each candidate is also known as the "composite (or sometimes compensatory) approach" (Salgado, Viswervaran \& Ones, 2001).

Were we in a situation of absolute selection, the obtained $T$ value would then be compared to some predetermined threshold $T^{*}$, and, if $T \geq T^{*}$, the candidate would be retained, independently of any other candidate. In our situation of relative selection, the $N$ candidates are judged not from a confrontation of their $T$ score with a target value, but by comparing it to the $T$ scores of all other $N-1$ candidates, then retaining the top $n$ values, for a selection ratio of $n / N$. In this case, the $C$ constant becomes immaterial and can be dropped. Moreover, though they are numerical and vary on a linear scale, the $X$ and $Y$ variables have arbitrary, ad hoc distribution parameters, depending both on the variables used and the group being measured, a fact which, at least, would unduly complicate the following treatment. To obviate this difficulty, we standardize each variable separately, through the $z$-score transformation, i.e.:

$$
x=\frac{X-\bar{X}}{s_{X}}, y=\frac{Y-\bar{Y}}{s_{Y}},
$$

each variable now having mean 0 and standard deviation 1 , and equation (3) will be rewritten as :

$$
t=b_{X} \cdot x+b_{Y} \cdot y \text {. }
$$

Setting also variable $t$ in standard form, with variance 1 , we may write:

$$
1=b_{X}^{2}+b_{Y}^{2}+2 \rho_{X, Y} b_{X} b_{Y}
$$

whence, for instance:

$$
b_{Y}=\sqrt{1-b_{X}^{2}\left(1-\rho_{X, Y}^{2}\right)}-b_{X} \rho_{X, Y} .
$$

Equation (7) leaves only coefficient $b_{X}$ to be determined for each specific situation.

The computed $t$ value is that by which the sorting of meritorious individuals is effected, and it is apparent that it is through linear coefficients $b_{X}$ and $b_{Y}$ that the relative importance, i.e. the weights $w_{X}$ and $w_{Y}$ assigned to criteria $X$ and $Y$, is to be conveyed. The question, now, is how do we fix coefficients $b_{X}$ and $b_{Y}$ so that the decreed, predetermined weights $w_{X}$ and $w_{Y}$ are complied with and reflect in the selection results, $D_{X}$ and $D_{Y}$. Expressed programmatically:

$$
\text { Set } b_{X} \text { and } b_{Y} \text { so that } D_{X}=w_{X} \text { and } D_{Y}=w_{Y} \text {. }
$$

The selection process is intrinsically complex, the more so if it is relative and its performance and output vary as a function of the selection ratio, a disadvantage which escapes absolute selection. For this reason, determination of coefficients $b_{X}$ and $b_{Y}$ cannot be rigorous, and we contemplated three solutions for carrying it out.

\section{Solution 1, Monte Carlo determination}

The simplest, although most laborious solution for determining the values of coefficients $b_{X}$ and $b_{Y}$, consists in running random Monte Carlo samples for some values of parameters $N, n$ and $\rho_{X, Y}$ varying coefficient $b_{X}$ (and its companion $b_{Y}$ ) in a purposeful manner, until the dominance index $D_{X}$ matches $w_{X}$ (approximately), with a corresponding match between $D_{Y}$ and $w_{Y}$. Monte Carlo simulation runs were effected using samples of pseudo random normal deviates. Table 2 presents the estimated $b_{X}$ coefficients ( $b_{Y}$ may be obtained by eq. 7), for various data configurations. Detailed information on the Monte Carlo simulation runs is given below table 2 . 
Table 2 - Estimated $b_{X}$ coefficients to be used with eq. 5 , as a function of the weight $\left(w_{X}\right)$ of the $X$ criterion, correlation $\left(\rho_{X, Y}\right)$ between criteria $X$ and $Y$, and selection rate $\left(\tau_{s}\right) \dagger$

\begin{tabular}{|c|c|c|c|c|c|}
\hline$\tau_{\mathrm{S}}$ & $\rho$ & $W_{X}=0.6$ & $W_{X}=0.7$ & $w_{X}=0.8$ & $W_{X}=0.9$ \\
\hline \multirow[t]{5}{*}{0.1} & 0 & 0.789 & 0.860 & 0.925 & 0.974 \\
\hline & 0.25 & 0.720 & 0.802 & 0.875 & 0.943 \\
\hline & 0.5 & 0.670 & 0.760 & 0.843 & 0.924 \\
\hline & 0.7 & 0.639 & 0.732 & 0.824 & 0.912 \\
\hline & 0.9 & 0.612 & 0.711 & 0.806 & 0.905 \\
\hline \multirow[t]{5}{*}{0.3} & 0 & 0.805 & 0.886 & 0.946 & 0.986 \\
\hline & 0.25 & 0.731 & 0.819 & 0.894 & 0.955 \\
\hline & 0.5 & 0.677 & 0.771 & 0.856 & 0.932 \\
\hline & 0.7 & 0.643 & 0.738 & 0.830 & 0.918 \\
\hline & 0.9 & 0.613 & 0.713 & 0.809 & 0.907 \\
\hline \multirow[t]{5}{*}{0.5} & 0 & 0.809 & 0.891 & 0.951 & 0.988 \\
\hline & 0.25 & 0.734 & 0.823 & 0.898 & 0.957 \\
\hline & 0.5 & 0.679 & 0.773 & 0.858 & 0.934 \\
\hline & 0.7 & 0.643 & 0.741 & 0.831 & 0.920 \\
\hline & 0.9 & 0.613 & 0.712 & 0.811 & 0.907 \\
\hline \multirow[t]{5}{*}{0.7} & 0 & 0.811 & 0.893 & 0.953 & 0.988 \\
\hline & 0.25 & 0.735 & 0.825 & 0.900 & 0.959 \\
\hline & 0.5 & 0.679 & 0.774 & 0.860 & 0.935 \\
\hline & 0.7 & 0.644 & 0.741 & 0.833 & 0.921 \\
\hline & 0.9 & 0.612 & 0.712 & 0.812 & 0.909 \\
\hline \multirow[t]{5}{*}{0.9} & 0 & 0.819 & 0.903 & 0.960 & 0.991 \\
\hline & 0.25 & 0.740 & 0.833 & 0.908 & 0.963 \\
\hline & 0.5 & 0.683 & 0.779 & 0.866 & 0.940 \\
\hline & 0.7 & 0.646 & 0.744 & 0.837 & 0.924 \\
\hline & 0.9 & 0.615 & 0.713 & 0.812 & 0.912 \\
\hline
\end{tabular}

† The Monte Carlo data samples were generated as $N \rho$-correlated $X, Y$ normal deviates, restandardized to mean 0 and variance 1 , and trial $b_{X}, b_{Y}$ values were used to compute the $t$ score (eq. 5 ), allowing the selection of $n=\tau_{S} \times \mathrm{N}$ candidates to obtain $D_{X}$. Corrections were then made to coefficients $b_{X}, b_{Y}$ and the convergent process iterated until $D_{X} \approx w_{X}$. For definiteness, we used $N=$ 1000 , such that, for instance, $\tau_{S}=0,3$ corresponded to $n=300$ people to be selected. The corresponding $b_{Y}$ coefficient is obtained by (7).

Linear coefficient $b_{X}$ increases trivially with the corresponding criterion's weight $w_{X}$, and it also shows a small but consistent increase with selection rate $\tau_{\mathrm{S}}$. Conversely, the positive correlation $\left(\rho_{X, Y}\right)$ between criteria brings about a reduction of the dominating coefficient $b_{X}$, which glides down toward its asymptotic value $w_{X}$. This could be expected by the fact that a positive $X-Y$ covariance increases the value of the $n_{X, Y}$ selection component, thus incorporating an excess of $Y$ individuals and concomitantly trimming down the $n_{*}, Y$ component, an effect which needs to be compensated by a relative reduction of $b_{X}$ and parallel increase of $b_{Y}$.

\section{Solution 2, By proportional contribution of variance}

The determining variation of the $t$ scores is traceable to contributions by both variables $x$ and $y$ and their correlation, as can be seen in expressions (5) and (6). Now, the separate contribution of, say, variable $x$ can be roughly identified with the variance of $x$, plus half the $x, y$-covariance term, obtaining $b_{X}{ }^{2}+\rho_{X, Y} b_{X} b_{Y}$. This leads to yet another proposition to fix the $b$ coefficients:

Set $b_{X}$ and $b_{Y}$ so that 
Table 3 - Computed $b_{X}$ coefficients based on expressions (10)-(11) to be used with eq. 5 , as a function of the weight $\left(w_{X}\right)$ of the $X$ criterion and correlation $\left(\rho_{X, Y}\right)$ between criteria $X$ and $Y$

\begin{tabular}{ccccc}
\hline$\rho$ & $w_{X}=0.6$ & $w_{X}=0.7$ & $W_{X}=0.8$ & $W_{X}=0.9$ \\
\hline 0 & 0.774 & 0.837 & 0.894 & 0.949 \\
0.25 & 0.709 & 0.781 & 0.851 & 0.921 \\
0.5 & 0.662 & 0.744 & 0.826 & 0.909 \\
0.7 & 0.633 & 0.723 & 0.813 & 0.904 \\
0.9 & 0.610 & 0.707 & 0.804 & 0.901 \\
\hline
\end{tabular}

$$
b_{X}^{2}+\rho_{X, Y} b_{X} b_{Y} \approx w_{X} \text { and } b_{Y}^{2}+\rho_{X, Y} b_{X} b_{Y} \approx w_{Y} .
$$

This type of analysis concurs with what is known in the literature as "effective weights", e.g. $b_{X}^{2}+\rho_{X, Y} b_{X} b_{Y}$, vs. "nominal weights", e.g. $b_{X}$ or $b_{X}^{2}$, determination (Wang \& Stanley, 1970; Kolen 2004; Guion, 2011), although the said literature stops there, confining itself to variance calculations and disregarding the actual, individual selection results (as mirrored in the $D_{X}$ and $D_{Y}$ statistics).

A solution for system (9) in two coefficients was found by Caroline Lambert ${ }^{3}$. Let $k$ and $a$ be :

$$
\begin{gathered}
k^{2}=\frac{1-\sqrt{1-\left(1-\rho_{\mathrm{X}, \mathrm{Y}}^{2}\right)\left[1+\left(1-2 \mathrm{w}_{X}\right)^{2} \times \rho_{X, Y}^{2}\right]}}{1-\rho_{X, Y}^{2}} ; \\
a=\frac{1}{2} \cos ^{-1}\left[\frac{2 \mathrm{w}_{X}}{k^{2}}\right]
\end{gathered}
$$

then :

$$
b_{X}=k \cos (a), b_{Y}=k \sin (a) .
$$

One may note that:

$$
\begin{gathered}
b_{X}=\sqrt{w_{X}}, b_{Y}=\sqrt{w_{Y}} \quad \text { if } \rho_{X, Y}=0, \\
b_{X} \rightarrow w_{X}, \text { and } b_{Y} \rightarrow w_{Y} \text { if } \rho_{X, Y} \rightarrow 1 .
\end{gathered}
$$

Table 3 presents values of coefficient $b_{X}$ obtained from (10)-(11) under conditions comparable to Table 2; note that the selection rate $\tau_{S}$ has no impact on these computations.

The reader may see that the pattern of coefficients

3 Caroline Lambert, Ph.D., Montréal, 2011, personal communication. in Table 3 mimics that of Table 2, at each level of selection rate $\left(\tau_{s}\right)$, but, being impervious to that parameter, the upward variation of $b_{X}$ as a function of $\tau_{\mathrm{S}}$ is overlooked. Above all, every coefficient in Table 3 lies below the corresponding value in Table 2, the gap diminishing somewhat as the selection rate increases. These numerous and occasionally important differences disqualify the simple variance- or 'effectiveweight' method for controlling the differential selection problem.

\section{Solution 3, Setting $\rho_{X, Y}=0$}

A third solution, that may be seen as legitimate under specific considerations, consists in applying to all cases the coefficients obtained for the case wherein $\rho_{X, Y}=0$, in Table $2^{4}$. Indeed, in frequently used selection settings where an ability criterion plus a personality component are used, Ones, Viswervaran and Schmindt (1993) report that the inter-criterion correlation is 0 or near to 0 . In addition to being handy, this ' 0 ' solution is tenable on statistical grounds. If $w_{X}>W_{Y}$, we necessarily have $b_{X}>b_{Y}$ and should consequently obtain $D_{X} \approx w_{X}>D_{Y} \approx$ $w_{Y}$. Keeping coefficients $b_{X}, b_{Y}$ unchanged while increasing $\rho_{X, Y}$ will step up the contribution of both criteria, but more so for the higher one, with the effect that $D_{X}>w_{X}$ and $D_{Y}<w_{Y}$. In words, setting coefficients $b_{X}$ and $b_{Y}$ for $\rho_{X, Y}=0$ insures that the direct (individual) contribution of each criterion is adequately controlled, whereafter the actual correlation between criteria 'naturally' enriches the import of the higher-weighted one. A significant counter-argument to the use of this solution is that, for situations where $\rho_{X, Y}>0$, the $w_{X}, w_{Y}$ weights are not directly mirrored in the actual $D_{X}, D_{Y}$

${ }^{4}$ Use of the $b_{X}$ and $b_{Y}$ coefficients obtained at $\rho=0$ for samples where $\rho>0$ entails, for the selection variable $t$, in (5), a variance of $1+2 \rho \cdot b_{X} \cdot b_{Y}$, i.e. $t$ being no more standardized to a variance of 1 . This has no adverse consequence here, in our context of relative selection, whereas it would have to be tackled with in the context of absolute selection.

The Quantitative Methods for Psychology 
Table 4 - Selection components and dominance index $D_{X}$ for the case where $w_{X}=0.6, w_{Y}=0.4, \tau_{S}=$ 0.1 and coefficients in (5) are set to $\rho_{X, Y}=0\left(b_{X}=0.789, b_{Y}=0.614\right)$.

\begin{tabular}{cccccc}
\hline$\rho_{X, Y}$ & $n_{*, *}$ & $n_{X^{*}}$ & $n_{*, Y}$ & $n_{X, Y}$ & $D_{X}$ \\
\hline 0 & 0.150 & 0.450 & 0.300 & 0.100 & 0.600 \\
0.25 & 0.081 & 0.495 & 0.230 & 0.194 & 0.683 \\
0.5 & 0.037 & 0.475 & 0.170 & 0.323 & 0.735 \\
0.7 & 0.015 & 0.395 & 0.123 & 0.467 & 0.762 \\
0.9 & 0.004 & 0.241 & 0.068 & 0.687 & 0.781 \\
\hline
\end{tabular}

indices. Table 4 gives an illustration for the case where $w_{X}=0.6, w_{Y}=0.4$ and $\tau_{\mathrm{S}}=0.1$, by breaking down the (averaged) selection results in their individual components.

\section{Complementary remarks}

Among the three solutions considered for implementing the additive scheme of relative selection, the most convincing one is obviously solution 1 , because it ensures the (approximate) equality between the $w_{X}, w_{Y}$ weights and the $D_{X}, D_{Y}$ statistics. Table 2 presents these coefficients for an interesting range of conditions, simple linear interpolation being sufficient to fill in the intervals (and using $b_{X}=w_{X}$ for $\rho_{X, Y}=1$ ).

Values of coefficients $b_{X}$ in Table 2 were obtained using samples of $N=1000$ pseudo candidates, instead of 10000 or more. Subsequent verifications with smaller samples have shown that the printed values hold up quite well, even for $N<15$, so that the user should feel confident in using them.

On the other hand, all simulations were performed in the normal, linear context, and some side experiments have shown us that, for instance, uniformly distributed data render slightly different results and coefficients. Be it for this reason or any other contextual aspect, a thorough validation procedure should always be carried out before submitting people's careers and lives to a selection process.

\section{Selection by sequential screening}

Relative selection on two criteria can also proceed sequentially, sorting out a subsample of candidates who perform best on a first selection variable ${ }^{5}$, then retaining among them those who perform best on the

${ }^{5}$ As a variant, selection on the first criterion, e.g. a honesty or criminal propensity score, could be absolute, by comparing the obtained score $(X)$ to some agreed upon threshold $\left(X^{*}\right)$, leaving plain, single-variable relative selection for the second phase of the process. second variable. A noteworthy advantage of this multistage selection strategy is its economy, i.e. screening out candidates on a first (sometimes cheaper) selection variable, leaving a reduced number for the final selection. To be specific, let $N$ be the initial number of candidates, and $n$, the number we wish to retain; $N_{X}$ is the number of candidates that are first selected by variable $X$, and $N_{Y}$, the number of candidates, taken from the previous list of $N_{X}$, that are finally retained by selection on the second variable, $Y$. We see here that $N_{Y}$ $=n$. This sequential, or hierarchical, scheme is akin to the multi-stage procedure, or the so-called "multiplehurdle" approach in its sequential form (the multiplehurdle approach can also be applied simultaneously, a candidate being retained if all his scores reach the corresponding criteria's thresholds). The study by Converse, Peterson and Griffith (2009) is a landmark for researches comparing the composite vs. multiplehurdle approaches in multi-criterion systems, although neither their methodology nor any that we could find explore our decreed nominal weights situation nor do they ascertain the correspondence between criteria's weights and individual selection results.

To get the gist of our hierarchical selection scheme, consider two extreme cases, one in which $N_{X}=n$, the other where $N_{X}=N$. In the first case, the uppermost $n$ candidates are chosen by the first criterion, $X$, leaving no hold, no room for maneuver to select by criterion $Y$ : such a case refers obviously to a $w_{X}=1, w_{Y}=0$ weight design. The opposite extreme case is one where $N_{X}=N$, $N>N_{Y}=n$ : all candidates are passed on by criterion $X$, and the whole burden of selection is left to criterion $Y$ : here, $w_{X}=0$ and $w_{Y}=1^{6}$. We thus see that, for this scheme, the operating parameter is $N_{X}$, parameters $N$

${ }^{6}$ These two extreme and theoretical cases would not automatically produce equivalent selection results, e.g., results where $D_{X}=1, D_{Y}=0$ for the first case. Partially random pairings of $(X, Y)$ values under $-1<\rho_{X, Y}<1$ would spuriously elect candidates in the 0 -weighted variable. 
Table 5 - Pre-selection ratio on first criterion $X\left(r_{X}\right)$ for various relative weights $w_{X}$ as a function of final selection ratio $\left(\tau_{\varsigma}\right)$ and correlation between criteria $X$ and $Y^{\dagger}$

\begin{tabular}{ccccccccccc}
\hline$\tau_{S}$ & $\rho_{X, Y}$ & $w_{X}=0.1$ & $w_{X}=0.2$ & $w_{X}=0.3$ & $w_{X}=0.4$ & $w_{X}=0.5$ & $w_{X}=0.6$ & $w_{X}=0.07$ & $w_{X}=0.8$ & $w_{X}=0.9$ \\
\cline { 2 - 10 } 0.1 & 0 & 0.630 & 0.500 & 0.421 & 0.363 & 0.316 & 0.275 & 0.238 & 0.200 & 0.159 \\
& 0.25 & 0.566 & 0.438 & 0.362 & 0.310 & 0.268 & 0.232 & 0.200 & 0.169 & 0.139 \\
& 0.50 & 0.470 & 0.358 & 0.296 & 0.253 & 0.220 & 0.193 & 0.169 & 0.147 & 0.125 \\
& 0.70 & 0.365 & 0.281 & 0.236 & 0.205 & 0.182 & 0.163 & 0.146 & 0.131 & 0.116 \\
& 0.90 & 0.227 & 0.187 & 0.166 & 0.151 & 0.140 & 0.130 & 0.122 & 0.115 & 0.108 \\
\hline \multirow{4}{*}{0.3} & 0.25 & 0.835 & 0.734 & 0.660 & 0.600 & 0.548 & 0.500 & 0.454 & 0.409 & 0.359 \\
& 0.50 & 0.794 & 0.689 & 0.616 & 0.558 & 0.510 & 0.467 & 0.427 & 0.387 & 0.347 \\
& 0.70 & 0.639 & 0.624 & 0.557 & 0.507 & 0.466 & 0.430 & 0.397 & 0.366 & 0.334 \\
& 0.90 & 0.496 & 0.441 & 0.497 & 0.457 & 0.424 & 0.396 & 0.372 & 0.348 & 0.325 \\
& 0 & 0.915 & 0.851 & 0.797 & 0.386 & 0.368 & 0.352 & 0.338 & 0.326 & 0.313 \\
\hline \multirow{4}{*}{0.5} & 0.25 & 0.893 & 0.824 & 0.770 & 0.724 & 0.684 & 0.667 & 0.627 & 0.588 & 0.546 \\
& 0.50 & 0.854 & 0.783 & 0.731 & 0.689 & 0.653 & 0.621 & 0.590 & 0.561 & 0.531 \\
& 0.70 & 0.799 & 0.732 & 0.686 & 0.650 & 0.620 & 0.594 & 0.570 & 0.547 & 0.524 \\
& 0.90 & 0.690 & 0.643 & 0.612 & 0.589 & 0.571 & 0.555 & 0.540 & 0.527 & 0.514 \\
\hline \multirow{4}{*}{0.7} & 0 & 0.960 & 0.925 & 0.894 & 0.865 & 0.837 & 0.810 & 0.783 & 0.756 & 0.729 \\
& 0.25 & 0.951 & 0.914 & 0.881 & 0.852 & 0.826 & 0.800 & 0.775 & 0.751 & 0.726 \\
& 0.50 & 0.934 & 0.893 & 0.861 & 0.834 & 0.810 & 0.787 & 0.765 & 0.744 & 0.722 \\
& 0.70 & 0.906 & 0.866 & 0.836 & 0.811 & 0.790 & 0.771 & 0.753 & 0.735 & 0.718 \\
& 0.90 & 0.843 & 0.810 & 0.787 & 0.771 & 0.756 & 0.744 & 0.733 & 0.722 & 0.711 \\
\hline \multirow{6}{*}{0.9} & 0 & 0.989 & 0.979 & 0.968 & 0.958 & 0.949 & 0.939 & 0.929 & 0.920 & 0.910 \\
& 0.25 & 0.988 & 0.976 & 0.966 & 0.956 & 0.947 & 0.937 & 0.928 & 0.919 & 0.909 \\
& 0.50 & 0.984 & 0.972 & 0.961 & 0.952 & 0.943 & 0.934 & 0.925 & 0.917 & 0.908 \\
& 0.70 & 0.978 & 0.965 & 0.954 & 0.945 & 0.937 & 0.929 & 0.922 & 0.915 & 0.907 \\
& 0.90 & 0.960 & 0.947 & 0.938 & 0.931 & 0.925 & 0.920 & 0.915 & 0.910 & 0.905 \\
\hline
\end{tabular}

† Values computed with formula (16) for $\rho_{X, Y}=0$ and estimated through Monte Carlo sampling for $\rho_{X, Y}>0$ (estimated standard deviation $\leq 0.001$ ).

and $n\left(=N_{Y}\right)$ being set in advance. The nearer $N_{X}$ comes to $n$, the more importance criterion $X$ will have, leaving only $\left(N-N_{X}\right)$ elements for criterion $Y$ to decide upon.

The hierarchical scheme of relative selection is essentially non-parametric, in the sense that it needs no population model to implement, normal or otherwise; only rank order information is used for variables $X$ and $Y$, and no score transformation is needed. The cases where $X$ and $Y$ are stochastically independent, and $\rho_{X, Y}$ $=0$, can be dealt with easily. For any $N, N_{X}, N_{Y}=n$ system, let all $N$ candidates first be ordered on their $X$ value; the concomitant list of $Y$ values appears as a random permutation, indeed is equivalent to a random permutation of numbers 1 to $N$. On this model, for $\rho_{X, Y}$ $=0$, a cursory analysis indicates that:

$$
\begin{gathered}
n_{X, Y}=N_{Y} ; \\
n_{X^{*}}=N_{Y} \cdot\left(N-N_{X}\right) / N_{X} ;
\end{gathered}
$$

$$
\begin{gathered}
n^{*, Y}=\left(N_{X}-N_{Y}\right) ; \\
n_{, * *}=\left(N-N_{X}\right)\left(N_{X}-N_{Y}\right) / N_{X} .
\end{gathered}
$$

Using equations (13) for supplementing values to components $n_{X^{*}}$ and $n^{*, Y}$ in (1), the dominance index becomes :

$$
D_{\mathbf{X}}=\frac{N_{Y} \cdot\left(N-N_{X}\right)}{N \cdot N_{Y}+N_{X} \cdot\left(N_{X}-2 N_{Y}\right)}
$$

Formula (14) can be recast in terms of pre-selection rate $r_{X}=N_{X} / N$ and global selection rate $\tau_{\mathrm{S}}=N_{Y} / N=n$ / $N$ :

$$
D_{\mathbf{X}}=\frac{\tau_{\mathrm{S}} \cdot\left(1-r_{X}\right)}{\tau_{\mathrm{S}}+r_{X} \cdot\left(r_{X}-2 \tau_{\mathrm{S}}\right)},
$$

from which we may derive : 


$$
r_{\mathbf{X}}=\frac{\tau_{x}}{2}\left(2-\frac{1}{D_{\mathbf{X}}}\right)+\sqrt{\tau_{x}^{2}\left(2-\frac{1}{D_{\mathbf{X}}}\right)-4 \tau_{x}\left(1-\frac{1}{D_{\mathbf{X}}}\right)}
$$

giving us the solution of the selection problem for $\rho_{X, Y}=$ 0 .

Introducing correlational dependency between $X$ and $Y$ complicates the situation somewhat, and we must again resort to Monte Carlo estimation for situations where $\rho_{X, Y}>0$. Table 5 presents values of $r_{X}$, the preselection rate, for a choice of conditions of $\tau_{\mathrm{S}}$ (the final selection rate), $\rho_{X, Y}$, and $W_{X}$, the weight decreed for criterion $X$. Use of the tabled coefficients ensures the user that the pre-determined weights $w_{X}$ and $W_{Y}$ imposed on his criteria are enforced adequately on the selection output.

\section{Summary comparison of the additive and sequential schemes of relative selection}

The additive and sequential schemes for relative selection presented here both ensure that the nominal, pre-determined weights assigned to the two criteria be obeyed, as reflected by the $D_{X}, D_{Y}$ dominance statistics. The seemingly similar situation in which two predictor variables are used to predict some outcome criterion variable by multiple regression analysis also involves weight coefficients, whether raw $(b)$ or standardized $(\beta)$, whose magnitudes reflect approximately the relative influence of the associated variable on the predicted value ${ }^{7}$. Although some parallelism exists between the two contexts, crucial differences remain. First, regression weights are aimed at controlling or predicting the value of some well-defined external outcome variable, whereas the weights operating in our two procedures were defined per se and based on a priori reasons, independently of any external criterion, and the procedure aimed at securing a close concordance between weight values and selection (retained / not retained) results. Second, regression analysis solutions and weight coefficients lean on variance control, in the same manner as does our additive procedure presented as Solution 2, a procedure that was discarded because of its inability to obtain the desired concordance.

Indeed, under conditions roughly similar to those implemented in this study, both selection schemes, additive and hierarchical, ensure that the criteria's respective weights $w_{X}$ and $w_{Y}$ are approximately echoed in the $D_{X}$ and $D_{Y}$ "dominance" statistics. But, do the two schemes select the same individuals, and how do they compare? Even though, logically, the same subgroup of meritorious individuals ought to be selected, the operational modes of the two schemes prevent a perfect match. Take, for instance, a situation where selection rate is low (say, $\tau_{\mathrm{S}} \approx 0.1$ ) and criterion $X$ is high-valued (say, $w_{X} \approx 0.9$ ). For the additive scheme, the two selection ingredients will be merged into a linear composite and processed for the full complement of candidates, so that every candidate will stand a fair chance to be selected thanks to his/her $X$ and $Y$ results. What happens in the hierarchical scheme is quite different: pre-selection on the $X$ criterion will be drastic (here, $r_{X} \approx 0.159-0.108$, see Table 5), so that a major part of candidates will be rejected, whatever their $Y$ results. Final selection will ensue on the basis of the $Y$ values of only the $X$ pre-selected individuals. Again through Monte Carlo sampling, we estimated the degree of concordance between the two schemes: Table 6 presents the percentage of co-selected individuals for an illustrative subset of conditions.

Concordance scores of selected individuals range from $68.2 \%$ to $97.8 \%$. As can be seen, the concordance between the additive and sequential schemes increases with increasing selection rate $\left(\tau_{S}\right)$ and with increasing correlation $\left(\rho_{X, Y}\right)$. There is also a somewhat subtle, parabolic effect of the $w_{X}, w_{Y}$ weights, concordance results showing a slight depression near the middle (0.5) weight values. It thus appears that, for some combinations of weights $\left(W_{X}, W_{Y}\right)$, selection rate $\left(\tau_{\mathrm{S}}\right)$ and correlation $\left(\rho_{X, Y}\right)$ conditions, a fair percentage of individuals rejected by one selection strategy would have been retained by the other.

\section{A worked out example}

To help recapitulate the proposed two selection procedures, here is a worked out example, schematically outlined. A firm needs to engage 45 new workers. 150 persons answered the job announcement, so that selection must be done. The chief of staff, in consultation with the company's management board, has decreed the use of two criteria, 'work ability' for $80 \%$, as measured through a 10 minute standardized work bout, and 'honesty' for $20 \%$, stemming from a standardized psychometric questionnaire ; correlation between the two measures is estimated null.

7 'Aliasing' effects can blur and even distort the true relative contributions of the predictor variables. 
Table 6 - Mean percentage of candidates picked out concomitantly by the additive and the hierarchical scheme of relative selection as a function of the global selection rate $\left(\tau_{S}\right)$, the weight of criterion $X\left(w_{X}\right)$ and the correlation between criteria $\left(\rho_{X, Y}\right)$

\begin{tabular}{ccrccrcrrr}
\hline & & $W_{X}=0.1$ & \multicolumn{3}{c}{$W_{X}=0.5$} & \multicolumn{3}{c}{$W_{X}=0.9$} \\
\hline$\tau_{\mathrm{S}}$ & $\rho_{X, Y}=0$ & 0.50 & 0.90 & $\rho_{X, Y}=0$ & 0.50 & 0.90 & $\rho_{X, Y}=0$ & 0.50 & 0.90 \\
0.1 & 74,6 & 86.6 & 94.8 & 68.2 & 79.0 & 90.6 & 69.0 & 80.1 & 91.4 \\
0.3 & 86.3 & 91.9 & 96.8 & 76.7 & 85.1 & 93.7 & 78.3 & 86.4 & 94.3 \\
0.5 & 91.8 & 94.8 & 97.8 & 82.8 & 89.3 & 95.6 & 84.6 & 90.4 & 96.1 \\
\hline
\end{tabular}

The preceding data correspond to $N=150$ candidates, an imposed selection ratio $\tau_{\mathrm{S}}$ of $45 / 150=$ 0.3 , pre-determined weights $w_{X}=0.8$ and $w_{Y}=0.2, \rho_{X, Y}$ $\approx 0$. In table 2 , we find $b_{X}=0.946$; by (7), we compute $b_{Y} \approx 0.324$. Hence, after separately standardizing i.e. converting to $z$-scores the 150 work and honesty measures for this group by (4), we calculate the weighted composite (5) for the additive procedure, i.e.:

$$
t=0.946 \times x+0.324 \times y,
$$

the 45 individuals with the highest $t$ values being selected. For the sequential, or two-stage procedure, Table 5 gives us $r_{X}=0.409$, so that we shall first preselect $r_{X} \times N=0.409 \times 150 \approx 61$ persons, those 61 obtaining the highest score on the $X$ (work ability) measure. Then, among those 61 persons only, we must find the 45 individuals having the highest result on the $Y$ (honesty) variable. The estimated commonality of candidates for this parameter set is $80 \%$, precisely $\sim 0.808 \pm 0,002$. This means that, on average, $0.808 \times$ $45 \approx 36$ individuals would be jointly retained by the additive and the hierarchical schemes, and 9 only by one and not the other.

\section{Conclusion}

Relative selection, a procedure by which a given number of individuals must be sorted out and retained from a larger pool of candidates, has been neglected in the literature, even if its practice is quite common in many industries and large companies. The case of selection on the basis of two promising, or theoretically profitable criteria, each with its own importance factor, has been minutely examined, and it was proven that, by appropriate procedures, the weights attributed to each criterion could be enforced and echoed in the selection results: in fact, a pair of weights like $\{80 \% ; 20 \%\}$ would be passed on the results, $80 \%$ of the differentially chosen candidates being ascribable to the first criterion, and $20 \%$ to the second. These nominally pre-weighted relative selection procedures, the additive and the sequential, can thus be added to the already long list of multi-criterion selection strategies (Sackett \& Roth, 1966; Guion, 2011; Ployhart, 2012).

Which of the two procedures is to be preferred? To answer this question, we must consider the whole personnel management situation, in both its financial aspect and its efficiency. The sequential, multi-stage scheme, in which part of the initial set of candidates is screened out by pre-selection on the first criterion, represents a financial and practical advantage, the more so if selection by the second criterion is costly to run or painstaking. Absolute prioritization of the first criterion is yet another argument in favour of this scheme: thanks to pre-selection, relatively unworthy candidates according to this criterion are sure to be rejected and cannot be 'score-compensated' through a two-variable composite. On the other hand, and when no criterion is to be prioritized over the other, the additive scheme, encompassing all candidates from the initial set, appears fairer, or more equitable, in that it uses all available information for every candidate and the selection decision is accountable to him for the two criteria. Finally, given that the criteria used for selection and their relative weights are agreed upon beforehand by all parties, the demonstrable and accountable correspondence between weights and actual selection results is an asset and a guarantee against quibble and judicial dispute.

Variants of our selection schemes can be thought up and have indeed been implemented and analysed (e.g. Sackett \& Roth, 1966), like one where pre-selection would be made with an absolute value-threshold for criterion 1, leaving simple relative selection for the remaining candidates for criterion 2. Relative selection systems using three criteria or more can also be implemented, and the methods developed and exemplified herein will hopefully inspire their designers. 


\section{References}

Barrett, G. V., Alexander, R. A., O'Connor, E. J., \& Forbes, J. B. (1978). Values and professional judgment in validating and litigating tests for civil service positions. Professional Psychology, 9, 137-144.

Converse, P. D., Peterson, M. H. \& Griffith, R. L. (2009). Faking on personality measures: Implications for selection involving multiple predictors. International Journal of Selection and Assessment, 17, 48-60.

Cronbach, L. J. (1971). Test validation, in R. L. Thorndike (ed.), Educational measurement (2 ${ }^{\text {nd }}$ ed.) (pp. 443-507). Washington, D.C.: American Council on Education.

Cronbach, L. J. (1990). Essentials of psychological testing ( $5^{\text {th }}$ ed.). New York: Harper \& Row.

Dawes, R. M., Corrigan, B. (1974). Linear models in decision making. Psychological Bulletin, 81, 95-106.

Deckro, R. F. (1977). M.B.A. admission criteria and academic success. Decision Sciences, 8, 765-769.

Dunnette, M. D. \& Borman, W. C. (1979). Personnel selection and classification. Annual Review of Psychology, 30, 477-525.

Guion, R. M. (2011). Assessment, measurement, and prediction ( $2^{\text {nd }}$ ed.). New York: Routledge.

Hodgkinson and J. K. Ford (eds.), International Review of Industrial and Organizational Psychology, vol. 27 (p. 153-196), Wiley-Blackwell.

Kane, M. T. (2006). Validation, in R. L. Brennon (ed.), Educational Measurement (4th ed.) (p. 17-64). Washington, D.C.: American Council on EducationPraeger.

Kolen, M. J., Brennan, R. L. (2004). Test equating, scaling, and linking: Methods and practices. New York, Springer.
Murphy, K. R., Shiarella, H. (1997). Implications of the multidimensional nature of job performance for the validity of selection tests: Multivariate frameworks for studying test validity. Personnel Psychology, 50, 823-854.

Ones, D. S., Viswervaran, C. \& Schmidt, F. L. (1993). Comprehensive meta-analysis of integrity test validities: Findings and implications for personnel selection and theories of job performance. Journal of Applied Psychology, 78, 679-703.

Ployhart, R. E. (2012). Personnel selection and the competitive advantage of firms, in G. P.

Society for Industrial and Organizational Psychogoly (SIOP) (1987). Principles for the validation and use of personnel selection procedures ( ${ }^{\text {rd }}$ ed.), Washington D.C.

Sackett, P. R. \& Roth, L. (1996). Multi-stage selection strategies: A Monte Carlo investigation of effects on performance and minority hiring. Personnel Psychology, 49, 549-472.

Salgado, J. F., Viswesvaran, C \& Ones, D. S. (2001). Predictors used for personnel selection: An overview of constructs, in N. Anderson, D. S. Ones, H. K. Sinangil \& C. Viswervaran (Eds): Handbook of industrial, word and organizational psychology (chap. 9, 165-199). SAGE publications.

Schmidt, F. L. \& Hunter, J. E. (1998). The validity and utility of selection methods in personnel psychology: Practical and theoretical implications of 85 years of research findings. Psychological Bulletin, 124, 262-274.

Wang, M. W., Stanley, J. C. (1970). Differential weighting: A review of methods and empirical studies. Review of Educational Research, 40, 663705.

\section{Citation}

Laurencelle, L. (2014). Relative selection as a function of two pre-weighted criteria: A study of two procedures. The Quantitative Methods for Psychology, 10 (1), 167-178.

\footnotetext{
Copyright (C) 2014 Laurencelle. This is an open-access article distributed under the terms of the Creative Commons Attribution License (CC BY). The use, distribution or reproduction in other forums is permitted, provided the original author(s) or licensor are credited and that the original publication in this journal is cited, in accordance with accepted academic practice. No use, distribution or reproduction is permitted which does not comply with these terms.
}

Received: 11/04/13 Accepted: 12/04/13 